\title{
Transitive Closure of Interval-valued Relations
}

\author{
Ramón González del Campo ${ }^{1}$ Luis Garmendia ${ }^{2} \quad$ Jordi Recasens $^{3}$ \\ 1.DSIC Universidad Complutense de Madrid \\ 2.DISIA Universidad Complutense de Madrid \\ 3.Universitat Politécnica de Catalunya \\ Email: rgonzale@estad.ucm.es, lgarmend@fdi.ucm.es,j.recasens@upc.edu
}

\begin{abstract}
This paper introduces some concepts of intervalvalued fuzzy relations and some of their properties: reflexivity, symmetry, T-transitivity, composition and locally reflexivity. It is also introduced the concept of T-transitive closure for an interval-valued fuzzy relation. An algorithm to compute the T-transitive closure of finite interval-valued relations, some properties and some examples are given.

Keywords - Generalized t-norms, Interval-valued Fuzzy Relations, Interval-valued Fuzzy Sets, t-norms, t-representable t-norms, t-transitive closure.
\end{abstract}

\section{Introduction}

Fuzzy sets, $\mathcal{F S}$, were introduced by Zadeh in 1.965 [1]. Since then many generalizations of fuzzy sets have been proposed to model the uncertainty and the vagueness in linguistic variables replacing the unit interval by another structure such as posets or lattices $[2,3,4]$. One of these generalizations are type-2 fuzzy sets, $\mathcal{F} \mathcal{S} 2,[5,6,7]$ were introduced by Zadeh. A Type2 fuzzy set on a universe of discourse $X, \mathcal{F} \mathcal{S} 2$, is a fuzzy set whose membership function is another fuzzy set on $[0,1]$ :

$$
A=\left\{\left((x, u), \mu_{A}(x, u)\right) \mid \forall x \in X, \forall u \in[0,1]\right\}
$$

Type- 2 fuzzy sets have been widely studied and applied since in many cases the uncertainty can be better expressed by a fuzzy set than by a single numeric value. The problem with type 2 fuzzy sets though is their computational complexity and the difficulty for an expert to select the adequate fuzzy subset as membership degree of an object to a linguistic label. This is why some simplifications have been proposed, such as the use of only some families of fuzzy sets such as triangular and trapezoidal ones. One of the most popular and useful simplification is the use of interval-valued fuzzy sets.

Interval-valued fuzzy sets $(\mathcal{I} \mathcal{V} \mathcal{F} \mathcal{S})$ were introduced in the 60s by Grattan-Guinness [8], Jahn [9], Sambuc [10] and Zadeh [5]. They are extensions of classical fuzzy sets where the membership value between 0 and 1 is replaced an interval in $[0,1]$. They easily allow to model uncertainty and vagueness because sometimes it is easier for experts to give a "membership interval" than a membership degree to objects on a universe. $\mathcal{I} \mathcal{V} \mathcal{F}$ are a special case of type-2 fuzzy sets that simplifies the calculations while preserving their richness as well. Intuitionistic fuzzy sets on $X(\mathcal{I} \mathcal{F} \mathcal{S})$ introduced by Atanassov [11]. In intuitionistic fuzzy sets each element has a membership degree, $\mu$, and a non-membership degree, $\nu$ satisfying: $\mu+\nu \leq 1$

$$
A=\{(x, \mu(x), \nu(x)) \mid x \in X, \mu(x), \nu(x) \in[0,1]\}
$$

The value $\pi=1-\mu-\nu$ is a measure of the uncertainty. This paper is organized as follows: In section three we introduce some important properties of interval-valued fuzzy relations such as reflexivity, symmetry, T-transitivity, composition and local reflexivity. Traditionally, the study of conjunctions between interval-valued fuzzy sets has been reduced to be modeled with t-representable t-norms. However, not all generalized t-norms are t-representable. Moreover, some of the non t-representable t-norms sometimes satisfy even more properties than t-representable t-norms [12]. Probably the most important property a fuzzy relation can fulfil is transitivity with respect a given t-norm. Since many times the data are given by a proximity relation $P$ (i.e.: a reflexive and symmetric but not necessarily transitive fuzzy relation), there are some methods to obtain a transitive relation close to $\mathrm{P}$ to replace it when transitivity is required. The most popular way to do this is calculating its transitive closure. In section four we introduce the concept of T-transitive closure for an interval-valued fuzzy relation and its expression in a finite universe for any t-norm. A few methods to compute it and some examples are given.

\section{Preliminaries}

\subsection{Interval-valued fuzzy sets}

Definition 2.1 [13] Let $\mathcal{L}^{I}=\left(L, \leq_{L}\right)$ be a lattice that satisfies:

1. $L=\left\{\left[x_{1}, x_{2}\right] \in[0,1]^{2}\right.$ with $\left.x_{1} \leq x_{2}\right\}$.

2. $\left[x_{1}, x_{2}\right] \leq_{L}\left[y_{1}, y_{2}\right]$ if and only if $x_{1} \leq y_{1}$ and $x_{2} \leq y_{2}$

Trivially:

$$
\begin{aligned}
& {\left[x_{1}, x_{2}\right]<_{L}\left[y_{1}, y_{2}\right] \Leftrightarrow x_{1}<y_{1}, x_{2} \leq y_{2} \text { or } x_{1} \leq} \\
& y_{1}, x_{2}<y_{2} \\
& {\left[x_{1}, x_{2}\right]={ }_{L}\left[y_{1}, y_{2}\right] \Leftrightarrow x_{1}=y_{1}, x_{2}=y_{2} .}
\end{aligned}
$$

$0_{L}={ }_{L}[0,0]$ and $1_{L}={ }_{L}[1,1]$ are the smallest and the greatest elements in $L$ respectively.

$\mathcal{L}^{I}$ is a complete lattice an the supremum and infimum are defined as follows.

Definition 2.2 [12] Let $\left\{\left[v_{i}, w_{i}\right]\right\}$ be a set of intervals on $L$. Then

1. Infimum: $\operatorname{Inf}_{L}\left\{\left[v_{i}, w_{i}\right]\right\} \equiv\left[\operatorname{infimun}\left\{v_{i}\right\}, \operatorname{infimun}\left\{w_{i}\right\}\right]$

2. Supremum: $\operatorname{Sup}_{L}\left\{\left[v_{i}, w_{i}\right]\right\} \equiv\left[\operatorname{supremun}\left\{v_{i}\right\}, \operatorname{supremun}\left\{w_{i}\right\}\right]$

Definition 2.3 [13] An interval-valued fuzzy set A on a universe $X$ is a mapping: 


$$
A=\left\{\left(a,\left[x_{1}, x_{2}\right]\right) \mid a \in X,\left[x_{1}, x_{2}\right] \in L\right\}
$$

Definition 2.4 [13] Let $X$ be a universe and $A$ and $B$ two interval-valued fuzzy sets. The equality between $A$ and $B$ is defined as: $A={ }_{L} B$ if and only if $A(a)={ }_{L} B(a) \forall a \in X$.

Definition 2.5 [13] Let $X$ be a universe and $A$ and $B$ two interval-valued fuzzy sets. The inclusion of $A$ in to $B$ is defined as: $A \subseteq_{L} B$ if and only if $A(a) \leq_{L} B(a) \forall a \in X$.

Definition 2.6 [13] A negation function for interval-valued fuzzy sets $\mathcal{N}$ is a decreasing function, $\mathcal{N}: L \rightarrow L$, that satisfies:

$$
\begin{aligned}
& \text { 1. } \mathcal{N}\left(0_{L}\right)={ }_{L} 1_{L} \\
& \text { 2. } \mathcal{N}\left(1_{L}\right)={ }_{L} 0_{L}
\end{aligned}
$$

If $\mathcal{N}\left(\mathcal{N}\left(\left[x_{1}, x_{2}\right]\right)\right)={ }_{L}\left[x_{1}, x_{2}\right]$ for all $\left[x_{1}, x_{2}\right]$ in $L$ then $\mathcal{N}$ is called an involutive negation.

Definition 2.7 A strong negation function for interval-valued fuzzy sets, $\mathcal{N}$, is a involutive function, $\mathcal{N}: L \rightarrow L$, that satisfies:

$$
\begin{aligned}
& \text { 1. } \mathcal{N}\left(0_{L}\right)={ }_{L} 1_{L} \\
& \text { 2. } \mathcal{N}\left(1_{L}\right)={ }_{L} 0_{L}
\end{aligned}
$$

T-norms are generalized to the lattice $\mathcal{L}^{I}$ in a straightforward way.

Definition 2.8 A generalized t-norm function [13], $\mathcal{T}$, is a monotone increasing, symmetric and associative operator, $\mathcal{T}$ : $L^{2} \rightarrow L$, that satisfies: $\mathcal{T}\left(1_{L},\left[x_{1}, x_{2}\right]\right)={ }_{L}\left[x_{1}, x_{2}\right]$ for all $\left[x_{1}, x_{2}\right]$ in $L$.

Due to monotony, it is easy to show:

$$
\mathcal{T}\left(\operatorname{Sup}_{L}\left\{\left[v_{i}, w_{i}\right]\right\},\left[y_{1}, y_{2}\right]\right) \geq_{L} \operatorname{Sup}_{L}\left\{\mathcal{T}\left(\left[v_{i}, w_{i}\right],\left[y_{1}, y_{2}\right]\right)\right\}
$$$$
\mathcal{T}\left(\operatorname{Inf}_{L}\left\{\left[v_{i}, w_{i}\right]\right\},\left[y_{1}, y_{2}\right]\right) \leq_{L} \operatorname{Inf}_{L}\left\{\mathcal{T}\left(\left[v_{i}, w_{i}\right],\left[y_{1}, y_{2}\right]\right)\right\}
$$

Due to the associativity of $\mathcal{T}$ the conjunction of three or more intervals can be defined inductively as:

$$
\begin{gathered}
\mathcal{T}(a, \mathcal{T}(b, c))={ }_{L} \mathcal{T}(\mathcal{T}(a, b), c)={ }_{L} a \Delta b \triangle c \text { where } \\
\triangle=_{L} \mathcal{T} .
\end{gathered}
$$

where $a={ }_{L}\left[a_{1}, a_{2}\right], b={ }_{L}\left[b_{1}, b_{2}\right]$ and $c={ }_{L}\left[c_{1}, c_{2}\right]$.

$T\left(\left[x_{1}, x_{2}\right],\left[y_{1}, y_{2}\right]\right)$ and $\overline{T\left(\left[x_{1}, x_{2}\right],\left[y_{1}, y_{2}\right]\right)}$ will denote the lower and the higher values of $T\left(\left[x_{1}, x_{2}\right],\left[y_{1}, y_{2}\right]\right)$.

Definition 2.9 [14] Let $\left\{x_{i}\right\}$ in [0,1]. A t-norm T in $([0,1], \leq$ ) is left-continuous if it satisfies:

$$
T\left(\operatorname{Sup} x_{i}, y\right)=\operatorname{Sup} T\left(x_{i}, y\right)
$$

Right-continuity can be defined in a similar way. This property is also called sup-preserving.

Definition 2.10 [13] A generalized t-norm operator $\mathcal{T}$ is $t$ representable in $\mathcal{L}^{I}$ if there are two t-norms: $T_{1}$ and $T_{2}$ $\left(T_{1}, T_{2}\right.$, in $\left.([0,1], \leq)\right)$ that satisfy:

$$
\mathcal{T}\left(\left[x_{1}, x_{2}\right],\left[y_{1}, y_{2}\right]\right)=_{L}\left[T_{1}\left(x_{1}, y_{1}\right), T_{2}\left(x_{2}, y_{2}\right)\right]
$$

where $T_{1}(v, w) \leq T_{2}(v, w) \forall v, w \in[0,1]$.

Let $x={ }_{L}\left[x_{1}, x_{2}\right]$ and $y={ }_{L}\left[y_{1}, y_{2}\right]$ be two intervals on $\mathrm{L}$ :

\section{Example 2.1}

$\operatorname{Inf}_{L}\left(\left\{\left[x_{1}, x_{2}\right],\left[y_{1}, y_{2}\right]\right\}\right)={ }_{L}\left[\min \left(x_{1}, y_{1}\right), \min \left(x_{2}, y_{2}\right)\right]$ is $t$-representable by means of $T=\min$ in $([0,1], \leq)$ :

Example 2.2 The following product generalized t-norm $*_{L}$ is t-representable:

$$
*_{L}\left(\left[x_{1}, x_{2}\right],\left[y_{1}, y_{2}\right]\right)={ }_{L}\left[x_{1} * y_{1}, x_{2} * y_{2}\right]
$$

Example 2.3 There are two generalizations of the Lukasiewicz t-norm [12]:

- $T_{w}\left(\left[x_{1}, x_{2}\right],\left[y_{1}, y_{2}\right]\right)={ }_{L}$ $\left[\max \left(0, x_{1}+y_{1}-1\right), \max \left(0, x_{2}+y_{2}-1\right)\right]$

- $T_{W}\left(\left[x_{1}, x_{2}\right],\left[y_{1}, y_{2}\right]\right)={ }_{L}$

$$
\left[\max \left(0, x_{1}+y_{1}-1\right), \max \left(0, x_{1}+y_{2}-1, x_{2}+y_{1}-1\right)\right]
$$

Note that $T_{w}$ is t-representable but $T_{W}$ is not.

Definition 2.11 [13] A generalized t-conorm $\mathcal{S}$ is an increasing, commutative, and associative operator $\mathcal{S}: L^{2} \rightarrow L$, that satisfies: $\mathcal{S}\left(0_{L},\left[x_{1}, x_{2}\right]\right)={ }_{L}\left[x_{1}, x_{2}\right]$ and $\mathcal{S}\left(1_{L},\left[x_{1}, x_{2}\right]\right)={ }_{L}$ $1_{L}$.

Due to the associativity of $\mathcal{S}$ we can write:

$\mathcal{S}(a, \mathcal{S}(b, c))={ }_{L} \mathcal{S}(\mathcal{S}(a, b), c)={ }_{L} a \nabla b \nabla c$ where $\nabla={ }_{L} \mathcal{S}$.

For example, $\mathcal{S}=\operatorname{Sup}_{L}$ is a generalized t-conorm.

In this paper the next definition is proposed.

Definition 2.12 Let $\left\{\left[v_{i}, w_{i}\right]\right\}$ be in L. A generalized t-norm operator $\mathcal{T}$ is left-continuous if and only if:

$\mathcal{T}\left(\operatorname{Sup}_{L}\left\{\left[v_{i}, w_{i}\right]\right\},\left[y_{1}, y_{2}\right]\right)={ }_{L} \operatorname{Sup}_{L}\left\{\mathcal{T}\left(\left[v_{i}, w_{i}\right],\left[y_{1}, y_{2}\right]\right)\right\}$

Right-continuity can be defined in a similar way. A t-norm, a negation and the dual t-conorm of $\mathcal{T}$ with respect to $\mathcal{N}$ is called a De Morgan triplet.

\section{Interval-valued fuzzy relations}

This section contains some definitions on interval-valued fuzzy relations that generalize the ones of fuzzy relations valued on $[0,1]$. In the next section, $\mathcal{T}$-transitive relations will be generated using the $S u p-\mathcal{T}$ product.

Definition 3.1 [15] Let $X_{1}$ and $X_{2}$ be two universes of discourse. An interval-valued fuzzy relation $R: X_{1} \times X_{2} \rightarrow L$ (where $\mathcal{L}^{I}=\left(L, \leq_{L}\right)$ is a lattice) is a mapping:

$$
R=\left\{((a, b),[x, y]) \mid a \in X_{1}, b \in X_{2},[x, y] \in L\right\}
$$

where $x=R(a, b)$ and $y=\overline{R(a, b)}$.

In the rest of the paper, it is assumed that $X_{1}=X_{2}=X$.

Definition 3.2 An interval-valued relation $R: X^{2} \rightarrow L$ is reflexive if:

$$
R(a, a)={ }_{L} 1_{L} \forall a \in X
$$


Definition 3.3 An interval-valued relation $R: X^{2} \rightarrow L$ is $\left[\alpha_{1}-\alpha_{2}\right]$-reflexive if:

$$
R(a, a) \geq_{L}\left[\alpha_{1}, \alpha_{2}\right] \forall a \in X
$$

where $\alpha_{1}, \alpha_{2} \in[0,1]$ and $\alpha_{1} \leq \alpha_{2}$.

Definition 3.4 An interval-valued relation $R: X^{2} \rightarrow L$ is symmetric if:

$$
R(a, b)={ }_{L} R(b, a) \forall a, b \in X
$$

Definition 3.5 Let $\mathcal{T}$ be a generalized t-norm operator and let $R$ interval-valued fuzzy relation on $X . R$ is $\mathcal{T}$-transitive if:

$$
\mathcal{T}(R(a, b), R(b, c)) \leq_{L} R(a, c) \forall a, b, c \in X
$$

Proposition 3.1 If $\mathcal{T}$ is t-representable with $T_{1}$ and $T_{2}(\mathcal{T}=$ $\left.\left[T_{1}, T_{2}\right]\right)$ then an interval-valued relation $R: X^{2} \rightarrow L$ is $\mathcal{T}$-transitive if and only if $\underline{R}$ is $T_{1}$-transitive and $\bar{R}$ is $T_{2}$ transitive:

Definition 3.6 Let $\mathcal{T}$ a generalized t-norm. Let $\mathcal{S}$ a generalized t-conorm from $\mathcal{S}$ and let be $\nabla$ the $n$-ary generalized t-conorm defined by associativity. Let $R_{1}$ and $R_{2}$ be two interval-valued fuzzy relations on a finite set $X=\left\{c_{1}, \ldots c_{m}\right\}$. The $\mathcal{S}-\mathcal{T}$-composition of $R_{1}$ and $R_{2}$ is defined as follows:

$$
\left(R_{1} \diamond_{(\mathcal{S} \mathcal{T})} R_{2}\right)\left(c_{q}, c_{r}\right)={ }_{L} \nabla c_{k} \in X \mathcal{T}\left(R_{1}\left(c_{q}, c_{k}\right), R_{2}\left(c_{k}, c_{r}\right)\right)
$$

where $\quad \nabla c_{k} \in X \mathcal{T}\left(R_{1}\left(c_{q}, c_{k}\right), R_{2}\left(c_{k}, c_{r}\right)\right) \quad={ }_{L}$ $\mathcal{T}\left(R_{1}\left(c_{q}, c_{1}\right), R_{2}\left(c_{1}, c_{r}\right)\right) \nabla \cdots \nabla \mathcal{T}\left(R_{1}\left(c_{q}, c_{m}\right), R_{2}\left(c_{m}, c_{r}\right)\right)$.

Definition 3.7 The local equality relation of a fuzzy intervalvalued fuzzy relation $R$ on $X$ is the fuzzy relation $E_{R}$ is:

$$
E_{R}(a, b)= \begin{cases}\operatorname{Sup}_{L} \forall c \in X \max (R(a, c), R(c, a)) & a=b ; \\ {[0,0],} & a \neq b .\end{cases}
$$

Definition 3.8 An interval-valued relation $R$ is locally reflexive if $E_{R} \subseteq L R$.

It is easy to prove that if $R$ is an reflexive interval-valued relation then $R$ is a locally reflexive interval-valued relation.

\section{$4 \mathcal{T}$-transitive closure for IVFSs}

In this section it is proved the existence of the $\mathcal{T}$-transitive closure of an interval valued fuzzy relation and its calculation via $\sup -\mathcal{T}$. The sup $-\mathcal{T}$ product is associative if $\mathcal{T}$ is left continuous of the universe is finite. Moreover, it is proved that the $\sup -\mathcal{T}$ product is continuous and so is the calculation of the $\mathcal{T}$-transitive closure for finite universes. An algorithm to calculate the $\mathcal{T}$-transitive closure is provided.

Definition 4.1 Let $\mathcal{T}$ be a generalized t-norm and let $R$ : $X^{2} \rightarrow L$ be an interval-valued relation on a finite universe $X$. The $\mathcal{T}$-transitive closure of $R$ is the relation $R^{\mathcal{T}}: X \times X \rightarrow L$ that satisfies:

1. $R \subseteq_{L} R^{\mathcal{T}}$.

2. If $R \subseteq{ }_{L} R^{\prime}$ and $R^{\prime}$ is $\mathcal{T}$-transitive then $R^{\mathcal{T}} \subseteq{ }_{L} R^{\prime}$.

Lemma 4.1 $R$ is $\mathcal{T}$-transitive if and only if $R \diamond_{S u_{L} T} R \subseteq_{L}$ $R$.

ISBN: 978-989-95079-6-8
Proof.

- $R$ is $\mathcal{T}$-transitive $\Rightarrow R \diamond_{S u_{L} T} R \subseteq_{L} R$ : $\left(R \diamond_{S_{u p} T} R\right)(a, b)={ }_{L}$ $\operatorname{Sup}_{L}{ }_{c \in X}\{\mathcal{T}(R(a, c), R(c, b))\} \leq_{L} R(a, b)$ due to $\mathcal{T}(R(a, c), R(c, b)) \leq_{L} R(a, b) \forall a, b, c \in X$

- $R \diamond_{S_{u p} T} R \subseteq_{L} R \Rightarrow R$ is $\mathcal{T}$-transitive: $\operatorname{Sup}_{L} d \in X\{\mathcal{T}(R(a, d), R(d, b))\} \leq_{L} R(a, b) \forall a, b \in X$ Then $\forall c \in X \mathcal{T}(R(a, c), R(c, b)) \leq_{L} R(a, b) \square$

Lemma 4.2 Let $R$ be an interval-valued relation. If $R^{\mathcal{T}}$ exists then it is unique.

Proof. Let $S_{1}$ and $S_{2}$ be two relations. If $S_{1}$ and $S_{2}$ are transitive closures of $R$ then according to definition 4.1: $S_{1} \subseteq_{L} S_{2}$ and $S_{2} \subseteq{ }_{L} S_{1}$, consequently $S_{1}={ }_{L} S_{2} \square$.

Lemma 4.3 Let $R$ be an interval-valued relation in a universe $X$ and an let $\mathcal{T}$ be an arbitrary generalized t-norm. Then the transitive closure of $R$ always exists.

Proof. Consider the set $\Omega_{R}$ of $\mathcal{T}$-transitive fuzzy relations containing $R$. Let us define the fuzzy relation $S_{R}^{*}(a, b)={ }_{L}$ In $f_{L ; S \in \Omega_{R}}\{S(a, b)\}$. We will prove that $S_{R}^{*}$ is $\mathcal{T}$-transitive. Due to monotony of generalized $\mathcal{T}$-norms it is obtained: $\mathcal{T}\left(S_{R}^{*}(a, b), S_{R}^{*}(b, c)\right)={ }_{L}$

$\mathcal{T}\left(\operatorname{Inf}_{L} S_{1} \in \Omega_{R}\left\{S_{1}(a, b)\right\}, \operatorname{Inf}_{L} S_{2} \in \Omega_{R}\left\{S_{2}(b, c)\right\}\right)$

$\leq_{L} \operatorname{Inf}_{L} S_{1} \in \Omega_{R} \operatorname{Inf}_{L} S_{2} \in \Omega_{R}\left\{\mathcal{T}\left(S_{1}(a, b), S_{2}(b, c)\right)\right\}$

$\leq_{L} \operatorname{Inf}_{L} S \in \Omega_{R}\{\mathcal{T}(S(a, b), S(b, c))\}={ }_{L} S_{R}^{*}(a, c) \square$

Proposition 4.1 Let $X$ be an arbitrary universe. Let $\left\{S_{1}, \ldots, S_{n}\right\}$ a set of interval-valued relations. If $\mathcal{T}$ is a leftcontinuous generalized $\mathcal{T}$-norm, then it satisfies:

$$
\begin{aligned}
& R \diamond_{S_{u p}} \mathcal{T}\left(\operatorname{Sup}_{L \forall i=1 . . n}\left\{S_{i}\right\}\right)={ }_{L} \\
& \operatorname{Sup}_{L \forall i=1 . . n}\left\{R \diamond_{\text {Sup }_{L} \mathcal{T}} S_{i}\right\}
\end{aligned}
$$

Proof.

$$
\begin{aligned}
& R \diamond_{\operatorname{Sup}_{L} \mathcal{T}}\left(\operatorname{Sup}_{L} \forall i=1 . . n\left\{S_{i}\right\}\right)(a, c)={ }_{L} \\
& \operatorname{Sup}_{L b \in X}\left\{\mathcal{T}\left(R(a, b), \operatorname{Sup}_{L \forall i=1 . . n}\left\{S_{i}(b, c)\right)\right\}\right. \\
& ={ }_{L} \operatorname{Sup}_{L \forall i=1 . . n} \operatorname{Sup}_{L} b \in X\left\{\mathcal{T}\left(R(a, b), S_{i}(b, c)\right)\right\} \\
& ={ }_{L}\left(\operatorname{Sup}_{L \forall i=1 . . n}\left\{R \diamond_{\text {Sup }_{L} \mathcal{T}} S_{i}\right\}\right)(a, c) \square
\end{aligned}
$$

Definition 4.2 Given a generalized t-norm $\mathcal{T}$, the $\mathcal{T}$-power $R^{(n)_{\mathcal{T}}}$ of a fuzzy relation $R$ on $X$ is recursively defined as follows:

1. $R^{(1) \mathcal{T}} \equiv R$

2. $R^{(n)_{\mathcal{T}}} \equiv R^{(n-1)_{\mathcal{T}}} \diamond_{\operatorname{Sup}_{L} \mathcal{T}} R$

Lemma 4.4 If $A \subseteq_{L} B$ then $A^{(k)} \subseteq_{L} B^{(k)}$

Proof.

Trivial due to monotony of $\mathcal{T} \square$.

Theorem 4.1 Let $X$ be an arbitrary universe and $\mathcal{T}$ a leftcontinuous t-norm. The transitive closure of $R$ is:

$$
R^{\mathcal{T}}={ }_{L} \operatorname{Sup}_{L \forall k \in \mathbb{N}}\left\{R^{(k)_{\mathcal{T}}}\right\}
$$

Proof.

Let $S=S u p_{L \forall k \in \mathbb{N}}\left\{R^{(k)_{\mathcal{T}}}\right\}$ be. By proposition 4.1: 


$$
S^{(2)_{\mathcal{T}}}={ }_{L}
$$

$\left(\operatorname{Sup}_{L \forall k \in \mathbb{N}}\left\{R^{(k)_{\mathcal{T}}}\right\}\right) \diamond_{\operatorname{Sup}_{L} \mathcal{T}}\left(\operatorname{Sup}_{L} \forall l \in \mathbb{N}\left\{R^{(l)_{\mathcal{T}}}\right\}\right)={ }_{L}$ $\operatorname{Sup}_{L \forall k, l \in \mathbb{N}}\left\{R^{(k+l)_{\mathcal{T}}}\right\}={ }_{L} \operatorname{Sup}_{L \forall m \in \mathbb{N} \backslash\{0,1\}}\left\{R^{(m)_{\mathcal{T}}}\right\} \subseteq S$

By lemma 4.1, it follows that $S$ is T-transitive.

Moreover, if $R \subseteq S^{\prime}$ and $S^{\prime}$ is T-transitive, then again by lemma 4.1 and 4.4 it holds that:

$$
R^{(2)_{\mathcal{T}}} \subseteq_{L} S^{\prime(2)_{\mathcal{T}}} \subseteq_{L} S^{\prime}, \ldots, R^{(k)_{\mathcal{T}}} \subseteq_{L} S^{\prime(k)_{\mathcal{T}}} \subseteq_{L} S^{\prime}
$$

hence $S \subseteq_{L} S^{\prime}$ and $R^{\mathcal{T}}={ }_{L} S \square$.

Lemma 4.5 Let $\mathcal{T}$ be any generalized $t$-norm, then:

$$
\begin{gathered}
\mathcal{T}\left(\left[x_{1}, x_{2}\right],\left[y_{1}, y_{2}\right]\right) \leq_{L} \operatorname{Inf}_{L}\left(\left[x_{1}, x_{2}\right],\left[y_{1}, y_{2}\right]\right) \\
\forall\left[x_{1}, x_{2}\right],\left[y_{1}, y_{2}\right] \in L
\end{gathered}
$$

\section{Proof.}

$\mathcal{T}\left(\left[x_{1}, x_{2}\right],\left[y_{1}, y_{2}\right]\right) \leq_{L} \mathcal{T}\left(\left[x_{1}, x_{2}\right], 1_{L}\right)=_{L}\left[x_{1}, x_{2}\right]$ $\Rightarrow\left[\mathcal{T}\left(\left[x_{1}, x_{2}\right],\left[y_{1}, y_{2}\right]\right), \overline{\mathcal{T}}\left(\left[x_{1}, x_{2}\right],\left[y_{1}, y_{2}\right]\right)\right] \leq_{L}\left[x_{1}, x_{2}\right]$ $\mathcal{T}\left(\left[\overline{\left.\left.x_{1}, x_{2}\right],\left[y_{1}, y_{2}\right]\right)} \quad{ }_{L} \quad \mathcal{T}\left(\left[y_{1}, y_{2}\right],\left[x_{1}, x_{2}\right]\right) \quad \leq_{L}\right.\right.$ $\mathcal{T}\left(\left[y_{1}, y_{2}\right], 1_{L}\right)={ }_{L}\left[y_{1}, y_{2}\right]$

$\Rightarrow\left[\underline{\mathcal{T}}\left(\left[x_{1}, x_{2}\right],\left[y_{1}, y_{2}\right]\right), \overline{\mathcal{T}}\left(\left[x_{1}, x_{2}\right],\left[y_{1}, y_{2}\right]\right)\right] \leq_{L}\left[y_{1}, y_{2}\right]$ Therefore:

$\mathcal{T}\left(\left[x_{1}, x_{2}\right],\left[y_{1}, y_{2}\right]\right) \leq \operatorname{minimum}\left[x_{1}, y_{1}\right]$

$\overline{\overline{\mathcal{T}\left(\left[x_{1}, x_{2}\right],\left[y_{1}, y_{2}\right]\right)}} \leq$ minimum $\left[x_{2}, y_{2}\right] \Rightarrow$

$\mathcal{T}\left(\left[x_{1}, x_{2}\right],\left[y_{1}, y_{2}\right]\right) \leq_{L} \operatorname{Inf}_{L}\left(\left[x_{1}, x_{2}\right],\left[y_{1}, y_{2}\right]\right) \square$

Lemma 4.6 Let $\mathcal{S}$ be any generalized $t$-conorm, then:

$$
\begin{gathered}
\mathcal{S}\left(\left[x_{1}, x_{2}\right],\left[y_{1}, y_{2}\right]\right) \geq_{L} \operatorname{Sup}_{L}\left(\left[x_{1}, x_{2}\right],\left[y_{1}, y_{2}\right]\right) \\
\forall\left[x_{1}, x_{2}\right],\left[y_{1}, y_{2}\right] \in L
\end{gathered}
$$

\section{Proof.}

$\mathcal{S}\left(\left[x_{1}, x_{2}\right],\left[y_{1}, y_{2}\right]\right) \geq_{L} \mathcal{S}\left(\left[x_{1}, x_{2}\right], 1_{L}\right)={ }_{L}\left[x_{1}, x_{2}\right]$

$\Rightarrow\left[\mathcal{S}\left(\left[x_{1}, x_{2}\right],\left[y_{1}, y_{2}\right]\right), \overline{\left.\mathcal{S}\left(\left[x_{1}, x_{2}\right],\left[y_{1}, y_{2}\right]\right)\right]} \geq_{L}\left[x_{1}, x_{2}\right]\right.$

$\mathcal{S}\left(\left[\overline{\left.\left.x_{1}, x_{2}\right],\left[y_{1}, y_{2}\right]\right)}={ }_{L} \quad \mathcal{S}\left(\left[y_{1}, y_{2}\right],\left[x_{1}, x_{2}\right]\right) \quad \geq_{L}\right.\right.$

$\mathcal{S}\left(\left[y_{1}, y_{2}\right], 1_{L}\right)=_{L}\left[y_{1}, y_{2}\right]$

$\Rightarrow\left[\mathcal{S}\left(\left[x_{1}, x_{2}\right],\left[y_{1}, y_{2}\right]\right), \overline{\left.\mathcal{S}\left(\left[x_{1}, x_{2}\right],\left[y_{1}, y_{2}\right]\right)\right]} \geq_{L}\left[y_{1}, y_{2}\right]\right.$

Therefore:

$\mathcal{S}\left(\left[x_{1}, x_{2}\right],\left[y_{1}, y_{2}\right]\right) \geq \operatorname{maximun}\left[x_{1}, y_{1}\right]$

$\overline{\overline{\mathcal{S}\left(\left[x_{1}, x_{2}\right],\left[y_{1}, y_{2}\right]\right)}} \geq \operatorname{maximun}\left[x_{2}, y_{2}\right] \Rightarrow$ $\mathcal{S}\left(\left[x_{1}, x_{2}\right],\left[y_{1}, y_{2}\right]\right) \geq_{L} \operatorname{Sup}_{L}\left(\left[x_{1}, x_{2}\right],\left[y_{1}, y_{2}\right]\right) \square$

Due associativity of $\mathcal{T}$ :

$$
\begin{gathered}
\mathcal{T}\left(\left[x_{1}, x_{2}\right], \mathcal{T}\left(\left[y_{1}, y_{2}\right],\left[z_{1}, z_{2}\right]\right)\right)={ }_{L} \\
\mathcal{T}\left(\mathcal{T}\left(\left[x_{1}, x_{2}\right],\left[y_{1}, y_{2}\right]\right),\left[z_{1}, z_{2}\right]\right)={ }_{L}\left[x_{1}, x_{2}\right] \triangle\left[y_{1}, y_{2}\right] \Delta \\
{\left[z_{1}, z_{2}\right]} \\
\operatorname{Inf}_{L}\left(\left[x_{1}, x_{2}\right], \operatorname{In} f_{L}\left(\left[y_{1}, y_{2}\right],\left[z_{1}, z_{2}\right]\right)\right)={ }_{L} \\
\left.\operatorname{Inf}_{L}\left(\operatorname{Inf}_{L}\left(\left[x_{1}, x_{2}\right],\left[y_{1}, y_{2}\right]\right),\left[z_{1}, z_{2}\right]\right)\right)={ }_{L}\left[x_{1}, x_{2}\right] \triangle_{L-i n f} \\
{\left[y_{1}, y_{2}\right] \triangle_{L-i n f}\left[z_{1}, z_{2}\right]}
\end{gathered}
$$

Lemma 4.7 Let $\mathcal{T}$ be an arbitrary generalized $t$-norm. Let $P$ be a path with a cycle:

$$
\begin{gathered}
P \equiv R\left(a, a_{1}\right) \Delta R\left(a_{1}, a_{2}\right) \Delta \ldots \Delta R\left(a_{t-1}, a_{t}\right) \Delta \\
R\left(a_{t}, c_{1}\right) \triangle R\left(c_{1}, c_{2}\right) \Delta \ldots \Delta R\left(c_{q}, a_{t}\right) \Delta R\left(a_{t}, a_{t+1}\right) \Delta \\
\ldots \Delta R\left(a_{k}, b\right)
\end{gathered}
$$

then

$$
\begin{gathered}
P \equiv R\left(a, a_{1}\right) \triangle R\left(a_{1}, a_{2}\right) \triangle \ldots \Delta R\left(a_{t-1}, a_{t}\right) \Delta \\
R\left(a_{t}, c_{1}\right) \triangle R\left(c_{1}, c_{2}\right) \triangle \ldots \Delta R\left(c_{q}, a_{t}\right) \Delta R\left(a_{t}, a_{t+1}\right) \Delta \\
\ldots \Delta R\left(a_{k}, b\right) \leq_{L} R\left(a, a_{1}\right) \triangle R\left(a_{1}, a_{2}\right) \triangle \ldots \Delta \\
R\left(a_{t-1}, a_{t}\right) \triangle R\left(a_{t}, a_{t+1}\right) \triangle \ldots \Delta R\left(a_{k}, b\right)
\end{gathered}
$$

Proof.

Trivial due to $\mathcal{T}\left(\left[x_{1}, x_{2}\right],\left[y_{1}, y_{2}\right]\right) \quad \leq_{L} \quad\left[y_{1}, y_{2}\right]$ forall $\left[x_{1}, x_{2}\right],\left[y_{1}, y_{2}\right]$ in $L$ and associativity of $\mathcal{T} \square$.

Theorem 4.2 Let $X$ be a finite universe with cardinality $n$. The transitive closure of $R, R^{\mathcal{T}}$, is:

$$
R^{\mathcal{T}}={ }_{L} \operatorname{Sup}_{L k=1 . . n}\left\{R^{(k)_{\mathcal{T}}}\right\}
$$

Proof.

$$
\begin{aligned}
& R^{(k)_{T}}(a, b)={ }_{L} \\
& ={ }_{L} \quad \operatorname{Sup}_{L} a_{1}, a_{2}, \ldots, a_{k-1} R\left(a, a_{1}\right) \Delta R\left(a_{1}, a_{2}\right) \triangle \\
& \text {.. } \triangle R\left(a_{k-1}, b\right) \leq_{L} \\
& ={ }_{L} \quad \operatorname{Sup}_{L} a_{1}, a_{2}, \ldots, a_{k-1} R\left(a, a_{1}\right) \quad \triangle_{I n f_{L}} \\
& R\left(a_{1}, a_{2}\right) \triangle_{I n f_{L}} \quad \cdots \triangle_{I n f_{L}} R\left(a_{k-1}, b\right) \text { according } \\
& \text { to lemma 4.5. }
\end{aligned}
$$

Now suppose $k>n$. Then $R^{(k)} \subseteq \subseteq_{L} R^{(n)}$ because it exists a cycle (lemma 4.7). Therefore, the cases $k>n$ need not be considered $\square$.

Corollary 4.1 Let $X$ be a finite universe with cardinality $n$. If $R$ is a locally reflexive relation, the transitive closure of $R$ is:

$$
R^{\mathcal{T}}={ }_{L} \operatorname{Sup}_{L k=1 . . n-1}\left\{R^{(k)_{\mathcal{T}}}\right\}
$$

\section{Proof}

If $R$ is a locally reflexive relation then $R(a, b) \leq_{L} R(a, a)$. Consider a cycle of length $n$, then its strength is:

$R^{(n)_{\mathcal{T}}}(a, a)={ }_{L}$

$$
\begin{aligned}
& ={ }_{L} \quad \operatorname{Sup}_{L} a_{1}, a_{2}, \ldots, a_{k-1} R\left(a, a_{1}\right) \quad \triangle R\left(a_{1}, a_{2}\right) \quad \triangle \\
& \ldots, \Delta R\left(a_{k-1}, a\right) \leq_{L} \\
& \operatorname{Sup}_{L} a_{1}, a_{2}, \ldots, a_{k-1} R\left(a, a_{1}\right) \quad \triangle_{I n f_{L}} \\
& R\left(a_{1}, a_{2}\right) \quad \triangle_{\text {Inf }_{L}} \quad \ldots \quad \Delta_{\operatorname{Inf}_{L}} \quad R\left(a_{k-1}, a\right) \quad \leq_{L} \\
& R(a, a) \square
\end{aligned}
$$

Theorem 4.3 Let $X$ be a finite universe with cardinality $n$. If $R$ is a reflexive fuzzy relation on $X$ then the transitive closure of $R$ is:

$$
R^{\mathcal{T}}={ }_{L} R^{(n-1)_{\mathcal{T}}}
$$

\section{Proof.}

$R(a, b)^{(k)_{\mathcal{T}}}={ }_{L}$

$$
\begin{aligned}
& ={ }_{L} \mathcal{T}\left(R(a, a), R^{(k)_{\mathcal{T}}}(a, b)\right) \\
& \leq_{L} \operatorname{Sup}_{L} \operatorname{coX}\left\{T\left(R(a, c), R^{(k)_{\mathcal{T}}}(c, b)\right)\right\} \\
& ={ }_{L} R^{(k+1)_{\mathcal{T}}}(a, b)
\end{aligned}
$$

Therefore $R(a, b)^{(k)_{\mathcal{T}}} \leq_{L} \quad R^{(k+1)_{\mathcal{T}}}(a, b) \Leftrightarrow R^{(k)_{\mathcal{T}}} \subseteq$ $R^{(k+1)_{\mathcal{T}}} \square$

Theorem 4.4 Let $\mathcal{T}$ be a t-representable t-norm $(\mathcal{T}=$ $\left.\left[T_{1}, T_{2}\right]\right)$ and let $R=[\underline{R}, \bar{R}]$ be a interval-valued relation. Then $R^{\mathcal{T}}=\left[\underline{R}^{T_{1}}, \bar{R}^{T_{2}}\right]$.

\section{Proof.}

$$
R^{(k)_{\mathcal{T}}}(a, b)={ }_{L}
$$




$$
\begin{aligned}
& ={ }_{L} \quad \operatorname{Sup}_{L a_{1}, a_{2}, \ldots, a_{k-1}} R\left(a, a_{1}\right) \quad \triangle R\left(a_{1}, a_{2}\right) \quad \triangle \\
& \ldots, \Delta\left(a_{k-1}, b\right) \\
& ={ }_{L} \operatorname{Sup}_{L} a_{1}, a_{2}, \ldots, a_{k-1} \underline{\left[R\left(a, a_{1}\right)\right.} \Delta_{1} \underline{R\left(a_{1}, a_{2}\right) \Delta_{1}} \\
& \ldots, \Delta_{1} \quad R\left(a_{k-1}, b\right), \overline{R\left(\overline{\left.a, a_{1}\right)}\right.} \Delta_{2} \quad \overline{R\left(a_{1}, a_{2}\right)} \quad \Delta_{2} \\
& \left.\ldots, \Delta_{2} \overline{R\left(a_{k-1}, b\right)}\right] \\
& ={ }_{L}\left[\max _{a_{1}, a_{2}, \ldots, a_{k-1}} R\left(a, a_{1}\right) \triangle_{1} \quad R\left(a_{1}, a_{2}\right) \quad \Delta_{1}\right. \\
& \ldots, \triangle_{1} R\left(a_{k-1}, b\right) \\
& \text {, } \max _{a_{1}, a_{2}, \ldots, a_{k-1}} \overline{R\left(a, a_{1}\right)} \triangle_{2} \overline{R\left(a_{1}, a_{2}\right)} \triangle_{2} \ldots, \triangle_{2} \\
& \left.\overline{R\left(a_{k-1}, b\right)}\right] \\
& =\left[\underline{R^{(k) T_{1}}(a, b)}, \overline{R^{(k)_{T_{2}}}(a, b)}\right]
\end{aligned}
$$

where $\Delta=\left[\Delta_{1}, \Delta_{2}\right]$. Then $\mathcal{T}=\left[T_{1}, T_{2}\right] \Rightarrow R^{\mathcal{T}}=$ $\left[\underline{R}^{T_{1}}, \bar{R}^{T_{2}}\right] \square$.

Definition 4.3 The distance $d$ of the supremum on $L$ is defined for all $x_{1}, x_{2}, y_{1}, y_{2} \in[0,1]$ by $d\left(\left[x_{1}, x_{2}\right],\left[y_{1}, y_{2}\right]\right)=$ $\max \left(\left|x_{1}-y_{1}\right|,\left|x_{2}-y_{2}\right|\right)$.

Definition 4.4 Let $R, S$ be two interval-valued fuzzy relatons on a set $X$. The distance $d$ between $R$ and $S$ us defined by:

$$
d(R, S)=\sup _{x, y \in X} d(R(x, y), S(x, y))
$$

Lemma $4.8 d$ is a distance on the set $R_{X}$ of interval-valued fuzzy relations on $X \square$.

\section{Proof.}

The supremum of distances is a distance.

Theorem 4.5 Let $T$ be a generalized continuous $t$-norm and $R_{X}$ the set of interval-valued fuzzy relations on $X . R_{X}$ with the sup- $\mathcal{T}$ product is an ordered topological semigroup.

\section{Proof.}

- Associativity is a straightforward exercise.

- The interval-valued

relation $E(x, y)=\left\{\begin{array}{ll}{[1,1],} & \mathrm{x}=\mathrm{y} ; \\ {[0,0],} & \text { otherwise. }\end{array}\right.$ is the identity element of $R_{X}$.

- Continuity: Since $T$ is defined on a compact set, it is uniformly continuous. Therefore:

$$
\begin{gathered}
\forall \epsilon>0, \exists \delta>0 \text { such that } \\
\forall m, n, m^{\prime}, n^{\prime}, a, b, a^{\prime}, b^{\prime} \in[0,1] \\
\max \left(|m-a|,\left|m^{\prime}-a^{\prime}\right|,|n-b|,\left|n^{\prime}-b^{\prime}\right|\right) \Rightarrow \\
\mid \mathcal{T}\left(\left[m, m^{\prime}\right],\left[n, n^{\prime}\right]\right)-\mathcal{T}\left(\left[a, a^{\prime}\right],\left[b, b^{\prime}\right]\right)<\epsilon\left({ }^{*}\right)
\end{gathered}
$$

We want to prove that given two interval-valued fuzzy relations $A, B \in R_{X}$ :

$$
\forall \epsilon>0, \exists \delta>0 \text { such that } \forall M, N \in R_{X}
$$

$$
\max (d(M, A), d(N, B))<\delta \Rightarrow d(M \circ N, A \circ B)<\epsilon
$$

Given $\epsilon$, we take $\delta>0$ satisfying (*). Then:

$$
\begin{gathered}
d(M \circ N, A \circ B)= \\
\sup _{x, y \in X} \mid \sup _{z \in X} \mathcal{T}(M(x, z), N(z, y))- \\
\sup _{z \in X} \mathcal{T}(A(x, z), B(z, y)) \mid \leq \\
\sup _{x, y \in X} \sup _{z \in X} \mid \mathcal{T}(M(x, z), N(z, y))- \\
\mathcal{T}(A(x, z), B(z, y)) \mid \leq \epsilon
\end{gathered}
$$

- Monotonicity is an immediate consequence of the monotonicity of $\mathcal{T} \square$.

Corollary 4.2 For any positive integer $n$ the map assigning to an interval-values fuzzy relation $M$ on a finite set its $n^{\text {th }}$ power is non-decreasing and continuous if the corresponding generalized t-norm $\mathcal{T}$ is continuous.

Corollary 4.3 If a generalized t-norm $\mathcal{T}$ is continuous, the map that assigns the $\mathcal{T}$-transitive closure to the intervalvalued fuzzy relations on a finite set is non-decreasing and continuous.

Theorem 4.6 Given a method to calculate the transitive closure of a fuzzy relation. Nevertheless, there are more effective methods and algorithms. For example it is possible to use an extension of the Floyd-Warshall algorithm [16]

\subsection{Algorithm to compute the transitive closure of an interval-valued fuzzy relation}

Let $R$ be an interval-valued relation on a finite universe $X$ with cardinality $n$ and let $\mathcal{T}$ be a generalized t-norm. It is possible to compute $R^{\mathcal{T}}$ using the next algorithm:

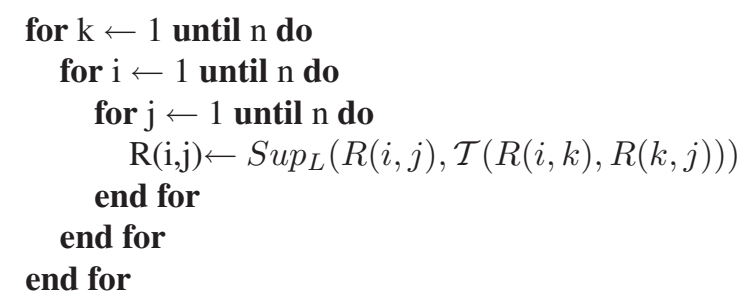

Example 4.1 Let $\mathcal{T}$ be $a$ generalized t-norm $T_{w}\left(\left[x_{1}, x_{2}\right],\left[y_{1}, y_{2}\right]\right)={ }_{L}\left[\max \left(0, x_{1}+\right.\right.$ $\left.\left.y_{1}-1\right), \max \left(0, x_{2}+y_{2}-1\right)\right]$ and let $R: X \times X \rightarrow L$ be the following interval-valued relation:

$$
R=\left(\begin{array}{cccc}
{[1,1]} & {[0.6,0.8]} & {[0.6,0.9]} & {[0,0]} \\
& {[1,1]} & {[0.4,0.9]} & {[0.6,0.9]} \\
& & {[1,1]} & {[0.6,0.9]} \\
& & & {[1,1]}
\end{array}\right)
$$

The computation of the $\mathcal{T}$-transitive closure of $R$ is the following:

$$
R^{\mathcal{T}}=\left(\begin{array}{cccc}
{[1,1]} & {[0.6,0.8]} & {[0.6,0.9]} & {[0.2,0.8]} \\
& {[1,1]} & {[0.4,0.9]} & {[0.6,0.9]} \\
& & {[1,1]} & {[0.6,0.9]} \\
& & & {[1,1]}
\end{array}\right)
$$

Note that by theorem $4.4, R^{\mathcal{T}}=\left[\underline{R^{W}}, \overline{R^{W}}\right]$

Example 4.2 Let $\mathcal{T}$ be $a$ generalized $t$ norm $T\left(\left[x_{1}, x_{2}\right],\left[y_{1}, y_{2}\right]\right)={ }_{L}\left[W\left(x_{1}, y_{1}\right), \operatorname{Min}\left(x_{2}, y_{2}\right)\right]$ and let $R: X \times X \rightarrow L$ be the following interval-valued relation:

$$
R=\left(\begin{array}{cccc}
{[1,1]} & {[0.6,0.8]} & {[0.6,0.9]} & {[0,0]} \\
& {[1,1]} & {[0.4,0.9]} & {[0.6,0.9]} \\
& & {[1,1]} & {[0.6,0.9]} \\
& & & {[1,1]}
\end{array}\right)
$$

The computation of the $\mathcal{T}$-transitive closure of $R$ is the following: 


$$
R^{\mathcal{T}}=\left(\begin{array}{cccc}
{[1,1]} & {[0.6,0.8]} & {[0.6,0.9]} & {[0.2,0.9]} \\
& {[1,1]} & {[0.4,0.9]} & {[0.6,0.9]} \\
& & {[1,1]} & {[0.6,0.9]} \\
& & & {[1,1]}
\end{array}\right)
$$

Note that by theorem $4.4, R^{\mathcal{T}}=\left[\underline{R^{W}}, \overline{R^{\text {Min }}}\right]$

Example 4.3 Let $\mathcal{T}$ be $a$ generalized t-norm $T_{W}\left(\left[x_{1}, x_{2}\right],\left[y_{1}, y_{2}\right]\right)={ }_{L}\left[\max \left(0, x_{1}+y_{1}-\right.\right.$ 1), $\left.\max \left(0, x_{1}+y_{2}-1, x_{2}+y_{1}-1\right)\right]$ and let $R: X \times X \rightarrow L$ be the following interval-valued relation:

$$
R=\left(\begin{array}{cccc}
{[1,1]} & {[0.6,0.8]} & {[0.6,0.9]} & {[0,0]} \\
& {[1,1]} & {[0.4,0.9]} & {[0.6,0.9]} \\
& & {[1,1]} & {[0.6,0.9]} \\
& & & {[1,1]}
\end{array}\right)
$$

The computation of the $\mathcal{T}$-transitive closure of $R$ is the following:

$$
R^{\mathcal{T}}=\left(\begin{array}{cccc}
{[1,1]} & {[0.6,0.8]} & {[0.6,0.9]} & {[0.2,0.5]} \\
& {[1,1]} & {[0.4,0.9]} & {[0.6,0.9]} \\
& & {[1,1]} & {[0.6,0.9]} \\
& & & {[1,1]}
\end{array}\right)
$$

Note that the generalized $\mathcal{T}$-norm in this example is not $t$ representable.

\section{An Application}

It is possible to model social relations using Interval-valued Fuzzy Sets. For example, it is possible to model "friendship". Let $R$ be a relation which describes the degree of friendship between pairs of people. If $A$ and $B$ are friends and $B$ and $C$ are friends, then it will probably exist some degree of friendship between $A$ and $C$. In this context, the $\mathcal{T}$-transitive closure can be used to learn friendship of individuals from a chain of friends, for example, from common friends.

Example 5.1 Let $R$ be an interval-valued relation which models the degree of friendship between four people $\{a, b, c, d\}$. It is possible to model the transitivity of friendship using the generalized t-norm $*_{L}\left(\left[x_{1}, x_{2}\right],\left[y_{1}, y_{2}\right]\right)={ }_{L}$ $\left[x_{1} * y_{1}, x_{2} * y_{2}\right]$ :

$$
R=\left(\begin{array}{cccc}
{[1,1]} & {[0.6,0.9]} & {[0.6,0.7]} & {[0,0]} \\
& {[1,1]} & {[0,0]} & {[0.2,0.8]} \\
& {[1,1]} & {[0,0]} \\
& & & {[1,1]}
\end{array}\right)
$$

Initially, $d$ has only friendship with $b$. In addition, the uncertainty of degree of friendship between $b$ and $d$ is wide. It is interesting to see how friendship is propagated.

$$
R^{\mathcal{T}}=\left(\begin{array}{cccc}
{[1,1]} & {[0.6,0.9]} & {[0.6,0.7]} & {[0.16,0.72]} \\
& {[1,1]} & {[0.48,0.63]} & {[0.2,0.8]} \\
& & {[1,1]} & {[0.096,0.5]} \\
& & & {[1,1]}
\end{array}\right)
$$

In $R^{\mathcal{T}} d$ has some degree of friendship with a, c learned by a common friend $b$. The uncertainty of degree of friendship of $d$ with the rest of them has been propagated as well.

\section{Conclusions}

Traditionally, the study of interval-valued fuzzy sets has been reduced to t-representable t-norms. We have generalized the main properties of interval-valued fuzzy relations: reflexivity, symmetry, composition, local reflexivity and t-transitivity. We also have generalized the T-transitive closure for any $\mathrm{t}$-norm and an algorithm to compute it is provided. It is analyzed the case of t-representable t-norms and proved that in this case the T-transitive closure of $R$ can be obtained from the two classical T-transitive closures of the lower and higher relations (theorem 4.4). Some examples are provided.

\section{References}

[1] L.A. Zadeh. Fuzzy sets. Information And Control, 8:338-353, 1965.

[2] H. Bustince, F. Herrera, and J. Montero. Fuzzy Sets and Their Extensions: Representation, Aggregation and Models, volume 220 of Studies in Fuzziness and Soft Computing. Hardcover, 2008.

[3] D. Gomez J. Montero and Bustince H. On the relevance of some families of fuzzy sets. Fuzzy Sets And Systems, 158:2429-2442, 2007.

[4] J. Goguen. L-fuzzy sets. Journal Of Mathematical Analysis And Applications, 18:145-174, 1967.

[5] L.A. Zadeh. The concept of a linguistic variable and its application to approximate reasoning i. Information Sciences, 8:199$249,1975$.

[6] L.A. Zadeh. The concept of a linguistic variable and its application to approximate reasoning ii. Information Sciences, 8:301-357, 1975.

[7] L.A. Zadeh. The concept of a linguistic variable and its application to approximate reasoning iii. Information Sciences, 9:43-80, 1975.

[8] I. Grattan-Guiness. Fuzzy membership mapped onto interval and many-valued quantities. Math. Logik. Grundladen Math, 22:149-160, 1975.

[9] K.U. Jahn. Intervall-wertige mengen. Math. Nach., 68:115132, 1975.

[10] E. Sanchez and R. Sambuc. Fuzzy relationships. phi -fuzzy functions. application to diagnostic aid in thyroid pathology. Proceedings of an International Symposium on Medical Data Processing, pages 513-524, 1976.

[11] K.T. Atanassov. Intuitionistic Fuzzy Sets. Physica-Verlag, Heidelberg, New York, 1999.

[12] C. Cornelis, G. Deschrijver, and E. Kerre. Advances and challenges in interval-valued fuzzy logic. Fuzzy Sets and Systems, 157(5):622-627, 2006.

[13] C. Cornelis, G.Deschrijver, and E. Kerre. Implication in intuitionistic fuzzy and interval-valued fuzzy set theory: construction, classification, application. Int. J. Approx. Reasoning, 35(1):55-95, 2004.

[14] B. De Baets and H. De Meyer. On the existence and construction of t-transitive closures. Inf. Sci., 152(1):167-179, 2003.

[15] H. Bustince and P. Burillo. Mathematical analysis of intervalvalued fuzzy relations: application to approximate reasoning. Fuzzy Sets Syst., 113(2):205-219, 2000.

[16] H. Naessens, H. De Meyer, and De B. Baets. Algorithms for the computation of t-transitive closures. IEEE Transactions on Fuzzy Systems, 10(1):541-551, 2002. 